\title{
An Obesity Risk SNP (rs17782313) near the MC4R Gene Is Associated with Cerebrocortical Insulin Resistance in Humans
}

\author{
Otto Tschritter, ${ }^{1}$ Axel Haupt, ${ }^{1,2}$ Hubert Preissl, ${ }^{3,4}$ Caroline Ketterer, ${ }^{1}$ Anita M. Hennige, ${ }^{1}$ \\ Tina Sartorius, ${ }^{1}$ Fausto Machicao, ${ }^{1}$ Andreas Fritsche, ${ }^{1}$ and Hans-Ulrich Häring ${ }^{1}$ \\ ${ }^{1}$ Department of Internal Medicine IV, University of Tübingen, Otfried-Müller-Strasse 10, 72076 Tübingen, Germany \\ ${ }^{2}$ Eli Lilly and Company, Lilly Deutschland GmbH, 61352 Bad Homburg, Germany \\ ${ }^{3}$ Institute of Medical Psychology and Behavioral Neurobiology, University of Tübingen, 72076 Tübingen, Germany \\ ${ }^{4}$ Department of Obstetrics and Gynecology, College of Medicine, University of Arkansas for Medical Sciences, \\ Little Rock, AR 72205, USA
}

Correspondence should be addressed to Otto Tschritter, otto.tschritter@med.uni-tuebingen.de

Received 30 November 2010; Revised 2 March 2011; Accepted 4 April 2011

Academic Editor: Jack A. Yanovski

Copyright (C) 2011 Otto Tschritter et al. This is an open access article distributed under the Creative Commons Attribution License, which permits unrestricted use, distribution, and reproduction in any medium, provided the original work is properly cited.

Activation of melanocortin-4 receptor (MC4R) by insulin sensitive neurons is a central mechanism in body weight regulation, and genetic variants in the MC4R gene (e.g., rs17782313) are associated with obesity. By using magnetoencephalography, we addressed whether rs 17782313 affects the cerebrocortical insulin response. We measured the cerebrocortical insulin response by using magnetoencephalography in a hyperinsulinemic euglycemic clamp (versus placebo) in 51 nondiabetic humans ( $26 \mathrm{f} / 25 \mathrm{~m}$, age $35 \pm 3$ years, BMI $28 \pm 1 \mathrm{~kg} / \mathrm{m}^{2}$ ). The C-allele of rs 17782313 was minor allele (frequency $23 \%$ ), and the genotype distribution (TT 30, TC 19, CC 2) was in Hardy-Weinberg-Equilibrium. Insulin-stimulated cerebrocortical theta activity was decreased in the presence of the C-allele (TT $33 \pm 16 \mathrm{fT}$; TC/CC $-27 \pm 20 \mathrm{fT} ; P=.023$ ), and this effect remained significant after adjusting for BMI and peripheral insulin sensitivity $(P=.047)$. Cerebrocortical theta activity was impaired in carriers of the obesity risk allele. Therefore, cerebral insulin resistance may contribute to the obesity effect of rs17782313.

\section{Introduction}

Melanocortin receptors (MC3R and MC4R) have been demonstrated in multiple brain regions including the hypothalamus $[1,2]$ and represent critical components of a regulating system for body weight and energy homeostasis. Both disruption of MC4R in mice [3] and mutations in the coding region of human $M C 4 R$ result in a severely obese phenotype $[4,5]$. Another relatively rare $(2-4 \%)$ polymorphism in the MC4R coding region has been reported to protect from obesity [6]. In recent genome-wide association studies (GWAS) also common genetic variants near the MC4R gene were associated with BMI [7], waist circumference, and insulin resistance [8].

Melanocortin receptors receive information from POMC and AgRP neurons about the nutritional and metabolic status. While POMC derivates like alpha-MSH and betaMSH stimulate melanocortin receptors, agouti-related protein (AgRP) is known to be a natural antagonist. As leptin and insulin activate POMC neurons and suppress AgRP neurons, both hormones contribute to the regulation of body weight and energy homeostasis via melanocortin receptors, and knock-out of MC4R results in decreased action of leptin and insulin in the brain [9].

We previously established a method to measure acute insulin responses in the human brain by combining magnetoencephalography (MEG) and the hyperinsulinemic euglycemic clamp technique [10]. In this study, we observed that cerebral insulin resistance is associated with obesity in humans and therefore speculated that a decreased insulin response of the brain might contribute to obesity caused by genetic alterations of MC4R. As rs17782313 had the strongest 
BMI signal in a GWAS study [7], we studied the effect of this single nucleotide polymorphism (SNP) on the insulin response of the brain.

\section{Methods}

2.1. Human Subjects and Experimental Design. We determined rs17782313 in the MC4R gene region in 51 subjects who were healthy by self-report and clinical examination and presented nondiabetic in an oral glucose tolerance test according to WHO/ADA criteria. Detailed characteristics of these subjects are given in Table 1.

2.2. Hyperinsulinemic Euglycemic Clamp and Saline Experiment with Measurement of Cerebrocortical Activity by Magnetoencephalography (MEG). To measure the insulin response of the brain, these subjects participated in an insulin and a placebo (=saline) experiment in random order on two different days approximately 1 to 2 weeks apart. Each experiment started at approximately 7.00 a.m. and consisted of a 30-minute baseline period, and a 2-step hyperinsulinemic euglycemic clamp or saline infusion. To maintain blood glucose at baseline levels a standard hyperinsulinemic euglycemic clamp protocol was followed. The details of the clamp procedures and the neurophysiologic measurements performed by MEG have been described in [10]. Here we used the change of spontaneous cortical beta and theta activity during insulin infusion (corrected for placebo derived changes) to quantify the cerebrocortical response to insulin. Beta and theta activity were extracted from spontaneous cortical activity by using fast Fourier transformation.

2.3. Analytical Procedures and Measurement of Body Fat. Plasma glucose was determined during the OGTT using the glucose oxidase method (YSI, Yellow Springs Instruments, Yellow Springs, CO, USA). Blood glucose was determined in the clamp experiments by a HemoCue blood glucose photometer (HemoCue AB, Aengelholm, Sweden). Plasma insulin levels were determined by microparticle enzyme immunoassay (Abbott Laboratories, Tokyo, Japan). Body composition was measured by bioelectrical impedance analysis (BIA-101A, RJL Systems, Detroit, Michigan, USA) and expressed as percent body fat.

2.4. Genotyping. For genotyping, DNA was isolated from whole blood using a commercial DNA isolation kit (NucleoSpin; Macherey \& Nagel, Düren, Germany). The SNPs were genotyped using the TaqMan assay (Applied Biosystems, Foster City, CA). The TaqMan genotyping reaction was amplified on a GeneAmp PCR system 7000 (Applied Biosystems) $\left(50^{\circ} \mathrm{C}\right.$ for 2 minutes, $95^{\circ} \mathrm{C}$ for 10 minutes, followed by 40 cycles of $95^{\circ} \mathrm{C}$ for 15 seconds and $60^{\circ} \mathrm{C}$ for 1 minute), and fluorescence was detected on an ABI Prism sequence detector (Applied Biosystems). Quality control was performed as reported in [11].

2.5. Statistical Analysis. For statistical analyses, MEG data of the saline experiment were subtracted from data of the
TABLE 1: Subject characteristics and effect of rs17782313 on obesity measures and peripheral insulin sensitivity.

\begin{tabular}{lccc}
\hline Genotype & TT & TC/CC & $P$ \\
\hline$N(\%)$ & $30(59 \%)$ & $21(41 \%)$ & - \\
Gender $(\mathrm{F} / \mathrm{M})$ & $14 / 16$ & $12 / 9$ & - \\
Age $($ years $)$ & $33 \pm 2$ & $38 \pm 3$ & .14 \\
Weight $(\mathrm{kg})$ & $81.4 \pm 3.4$ & $83.9 \pm 3.6$ & $.47^{*}$ \\
BMI $\left(\mathrm{kg} / \mathrm{m}^{2}\right)$ & $27.1 \pm 0.9$ & $28.4 \pm 1.0$ & $.62^{*}$ \\
$\begin{array}{l}\text { Body fat }(\%) \\
\text { Waist circumference }\end{array}$ & $27.1 \pm 1.6$ & $30.5 \pm 1.9$ & $.57^{*}$ \\
$\begin{array}{l}\text { (cm) } \\
\text { Fasting plasma glucose }\end{array}$ & $92 \pm 3$ & $95 \pm 3$ & $.34^{*}$ \\
$\begin{array}{l}\text { (mmol/L) } \\
\text { Hr glucose }\end{array}$ & $4.9 \pm 0.1$ & $5.1 \pm 0.1$ & $.35^{*}$ \\
$\begin{array}{l}\text { Inmol/L) } \\
(\mu \text { molin sensitivity index }\end{array}$ & $0.074 \pm 0.009$ & $0.062 \pm 0.010$ & $.67^{*}$ \\
\hline
\end{tabular}

$\mathrm{M} \pm \mathrm{SE}$; ${ }^{*}$ adjusted for age and gender; ${ }^{* *}$ OGTT; ${ }^{* * *}$ Hyperinsulinemic euglycemic clamp.

insulin experiment to correct for potential placebo effects and daytime variation. The change of the investigated MEG parameters from basal to the second step of the clamp was considered to indicate the total insulin effect and was used in further analyses.

Unless otherwise stated, data are means \pm SE. Nonnormally distributed variables (Shapiro-Wilk W test) were logarithmically transformed. To adjust for covariates and to identify independent relationships, we performed linear multiple regression analyses. In general, a $P$ value of $<.05$ was considered to indicate statistical significance. As we studied insulin-induced changes in beta and theta activity, correction for multiple comparisons requires an alpha level of 0.025 instead of 0.05 for the primary analysis of MEG data. Due to the very low number of CC carriers, determination of a per allele risk as suggested by another study [7] was not possible. Therefore, TC and CC carriers were treated as one group and compared with wild-type carriers in all further analyses. For all statistical analyses, the software package JMP (SAS Institute, Cary, NC) was used.

2.6. Ethical Statement. The study protocol was approved by the Ethical Committee of the University of Tübingen and informed written consent had been obtained from each subject prior to the studies. The whole study protocol has been designed in accordance with the declaration of Helsinki (1964). Furthermore, we certify that all applicable institutional and governmental regulations concerning the ethical use of human volunteers were followed during the research.

\section{Results}

3.1. Genotype Data and Body Weight Effect of rs17782313. The genotype distribution (TT 30, TC 19, and CC 2) was in Hardy-Weinberg-Equilibrium, and the minor C-allele had a frequency of $23 \%$. Carriers of the rare allele were 


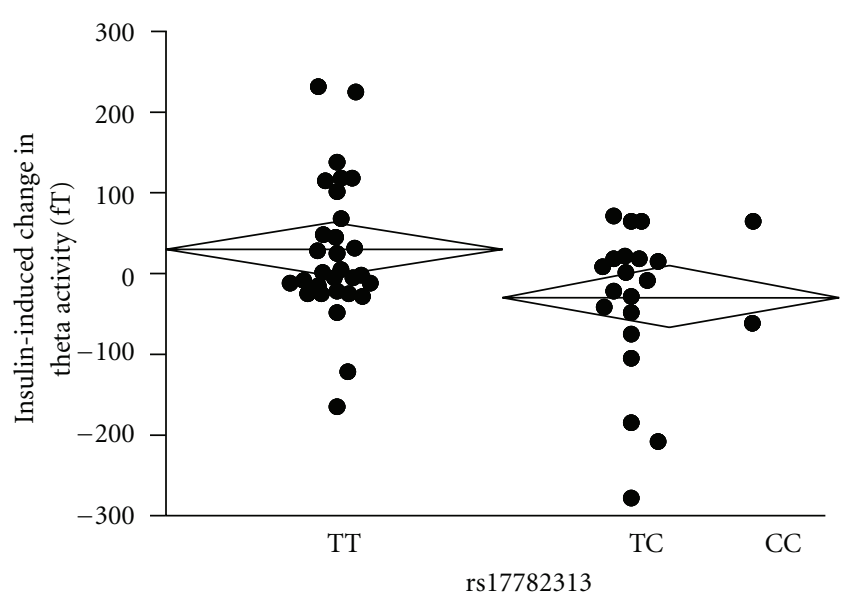

FIGURE 1: Insulin effect on theta activity in the genotype groups: in subjects carrying the rare allele of rs17782313 in the homozygous (TC) or the heterozygous form (CC), the effect of insulin on theta activity was significantly reduced as compared to carriers of the wild-type allele (TT) $(P=.023)$. The diamonds show the means of each group (horizontal line) and the confidence intervals of the means (upper and lower point). The effect of the genotype remained significant after adjusting for $\operatorname{BMI}(P=.038)$ and also after adjusting for BMI and peripheral insulin sensitivity $(P=.047)$.

$2.5 \mathrm{~kg}$ heavier, displayed a slightly increased BMI, waist circumference, and body fat as compared to the wild-type carriers; however, all these effects failed statistical significance (Table 1). According to the weight effect, peripheral insulin sensitivity was reduced in CC carriers.

3.2. Cerebrocortical Insulin Resistance in Carriers of rs17782313. Regarding the effect of insulin on the cerebral cortex, we observed a decreased effect on theta activity (Figure 1) in presence of the C-allele (TT $33 \pm 16 \mathrm{fT}$, TC/CC $-27 \pm 20 \mathrm{fT}, P=.023)$. This association remained significant after adjusting for BMI $(P=.038)$ and also after adjusting for BMI and peripheral insulin sensitivity $(P=.047)$. In contrast to theta activity, there was no significant reduction of insulin-mediated beta activity by rs17782313 (TT $3.0 \pm 3.2$ fT, TC/CC $-1.3 \pm 4.1$ fT, $P=.41)$.

\section{Discussion}

Our data demonstrate that the insulin response of the brain measured by magnetoencephalography (MEG) is diminished in carriers of rs17782313. From a mechanistical point of view, these data support the idea that a decreased insulin response of the brain is a pathogenic factor that contributes to the effect of this polymorphism on obesity. However, association data have some limitations, as they cannot definitely prove the direction of an effect and provide only indirect information on the underlying mechanisms. Here, it is clear that the primary site of the obesity effect must be located in the brain, as MC4R is highly expressed in the brain but not in peripheral tissues [1]. To get the best possible information from our data, we performed multiple regression models to adjust the cerebral effect of the polymorphism for obesity and for peripheral insulin resistance. As the SNP effect on the cerebrocortical insulin response remained significant, our data suggest that rs17782313 directly alters the insulin response of the brain.

In contrast to the cerebrocortical effect, the differences in obesity measures and metabolic parameters between the genotype group were not significant, though the effect size seems comparable to previous reports on MC4R polymorphisms $[7,8]$. One explanation for the lack of significance in these parameters is that altered brain function is likely to be the primary effect of the MC4R SNP, while obesity and metabolic alterations are secondary. Furthermore, BMI and waist circumference have limited accuracy in small sample sizes.

We have previously demonstrated reduced insulin responses of beta and theta activities in obese humans [10]. Notably, the effect of rs17782313 occurred only in the theta activity band, while there was no effect on beta activity. In contrast, we observed that variation in the FTO and IRS1 gene is associated with a reduction of insulin-mediated beta activity, while theta activity is not affected [10, 12]. Furthermore, we reported that beta and theta activities are differently affected by aging [13] and suggested that these parameters may represent different brain structures and functions and their responses to insulin. Therefore, these data suggest that suppressed cerebral insulin effects by genetic variation in FTO and aging involve different mechanisms than variation in the $M C 4 R$ gene.

It is interesting to note, that theta activity is mainly generated in the hippocampus [14]. Furthermore, the hippocampus has been shown to contribute to food-related reward in obese women [15]. Additionally, theta activity has been attributed to locomotor activity and voluntary movements [16]. Therefore, a decreased response of theta activity to insulin by the obesity risk allele of rs17782313 may indicate increased food reward and decreased locomotor activity as possible mechanisms that contribute to the development of obesity in these subjects.

\section{Conclusions}

Genetic variation in $M C 4 R$ leads to an impaired insulin response in theta activity in the human brain. Though specific suggestions on underlying brain functions are speculative, our data support that these functions may be different from alterations observed with aging and variation in the FTO gene.

\section{Acknowledgments}

The authors gratefully acknowledge the superb technical assistance of Anna Teigeler, Heike Luz, and Gabi Walker. This study was supported by the Deutsche Forschungsgemeinschaft (KFO 114 and FR 1561/4-1) and by the German Federal Ministry of Education and Research in the form of grants to the German Center for Diabetes Research (DZD e.V.) and to the Kompetenznetzwerk Adipositas 01GI0849. 


\section{References}

[1] K. G. Mountjoy, M. T. Mortrud, M. J. Low, R. B. Simerly, and R. D. Cone, "Localization of the melanocortin-4 receptor (MC4-R) in neuroendocrine and autonomic control circuits in the brain," Molecular Endocrinology, vol. 8, no. 10, pp. 1298 1308, 1994.

[2] L. Roselli-Rehfuss, K. G. Mountjoy, L. S. Robbins et al., "Identification of a receptor for gamma melanotropin and other proopiomelanocortin peptides in the hypothalamus and limbic system," Proceedings of the National Academy of Sciences of the United States of America, vol. 90, pp. 8856-8860, 1993.

[3] D. Huszar, C. A. Lynch, V. Fairchild-Huntress et al., "Targeted disruption of the melanocortin-4 receptor results in obesity in mice," Cell, vol. 88, no. 1, pp. 131-141, 1997.

[4] G. S. Yeo, I. S. Farooqi, S. Aminian, D. J. Halsall, R. G. Stanhope, and S. O'Rahilly, "A frameshift mutation in MC4R associated with dominantly inherited human obesity," Nature Genetics, vol. 20, no. 2, pp. 111-112, 1998.

[5] C. Vaisse, K. Clement, B. Guy-Grand, and P. Froguel, "A frameshift mutation in human MC4R is associated with a dominant form of obesity," Nature Genetics, vol. 20, no. 2, pp. 113-114, 1998.

[6] E. H. Young, N. J. Wareham, S. Farooqi et al., "The V103I polymorphism of the MC4R gene and obesity: population based studies and meta-analysis of 29563 individuals," International Journal of Obesity, vol. 31, no. 9, pp. 1437-1441, 2007.

[7] R. J. Loos, C. M. Lindgren, S. Li et al., "Common variants near MC4R are associated with fat mass, weight and risk of obesity," Nature Genetics, vol. 40, no. 6, pp. 768-775, 2008.

[8] J. C. Chambers, P. Elliott, D. Zabaneh et al., "Common genetic variation near MC4R is associated with waist circumference and insulin resistance," Nature Genetics, vol. 40, no. 6, pp. 716$718,2008$.

[9] M. W. Schwartz, S. C. Woods, D. Porte Jr., R. J. Seeley, and D. G. Baskin, "Central nervous system control of food intake," Nature, vol. 404, no. 6778, pp. 661-671, 2000.

[10] O. Tschritter, H. Preissl, A. M. Hennige et al., "The cerebrocortical response to hyperinsulinemia is reduced in overweight humans: a magnetoencephalographic study," Proceedings of the National Academy of Sciences of the United States of America, vol. 103, no. 32, pp. 12103-12108, 2006.

[11] N. Stefan, F. Machicao, H. Staiger et al., "Polymorphisms in the gene encoding adiponectin receptor 1 are associated with insulin resistance and high liver fat," Diabetologia, vol. 48, no. 11, pp. 2282-2291, 2005.

[12] O. Tschritter, H. Preissl, Y. Yokoyama, F. Machicao, H. U. Haring, and A. Fritsche, "Variation in the FTO gene locus is associated with cerebrocortical insulin resistance in humans," Diabetologia, vol. 50, no. 12, pp. 2602-2603, 2007.

[13] O. Tschritter, A. M. Hennige, H. Preissl et al., "Insulin effects on beta and theta activity in the human brain are differentially affected by ageing," Diabetologia, vol. 52, no. 1, pp. 169-171, 2009.

[14] C. D. Tesche and J. Karhu, "Theta oscillations index human hippocampal activation during a working memory task," Proceedings of the National Academy of Sciences of the United States of America, vol. 97, no. 2, pp. 919-924, 2000.

[15] L. E. Stoeckel, R. E. Weller, E. W. Cook III, D. B. Twieg, R. C. Knowlton, and J. E. Cox, "Widespread reward-system activation in obese women in response to pictures of highcalorie foods," Neuroimage, vol. 41, no. 2, pp. 636-647, 2008.
[16] E. Dzoljic, R. van Leeuwen, R. de Vries, and M. R. Dzoljic, "Vigilance and EEG power in rats: effects of potent inhibitors of the neuronal nitric oxide synthase," Naunyn-Schmiedeberg's Archives of Pharmacology, vol. 356, no. 1, pp. 56-61, 1997. 


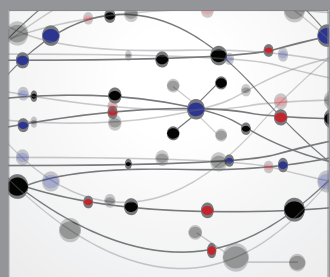

The Scientific World Journal
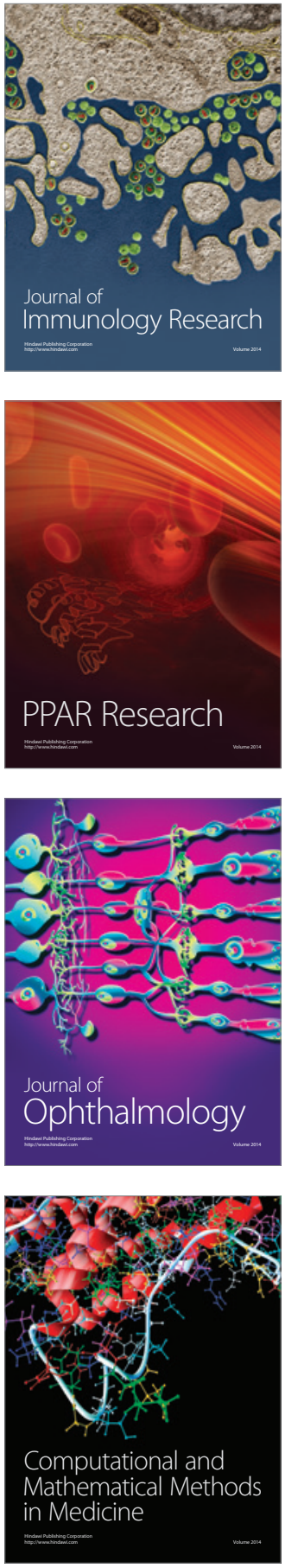

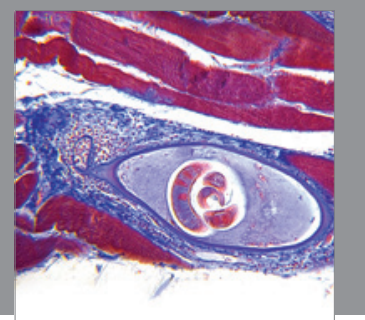

Gastroenterology

Research and Practice
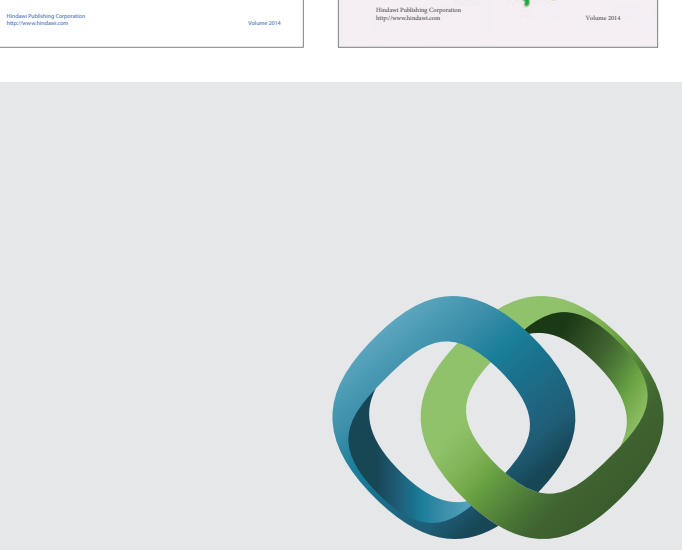

\section{Hindawi}

Submit your manuscripts at

http://www.hindawi.com
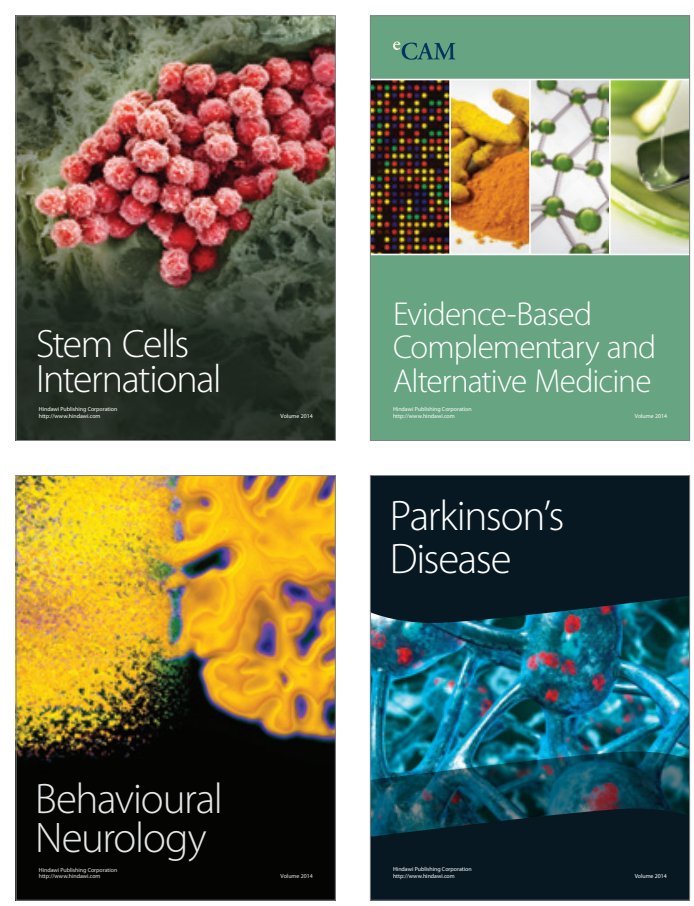

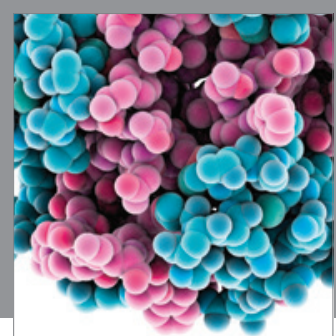

Journal of
Diabetes Research

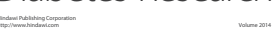

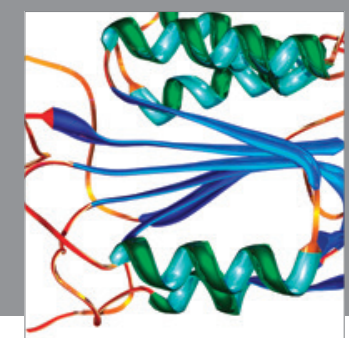

Disease Markers
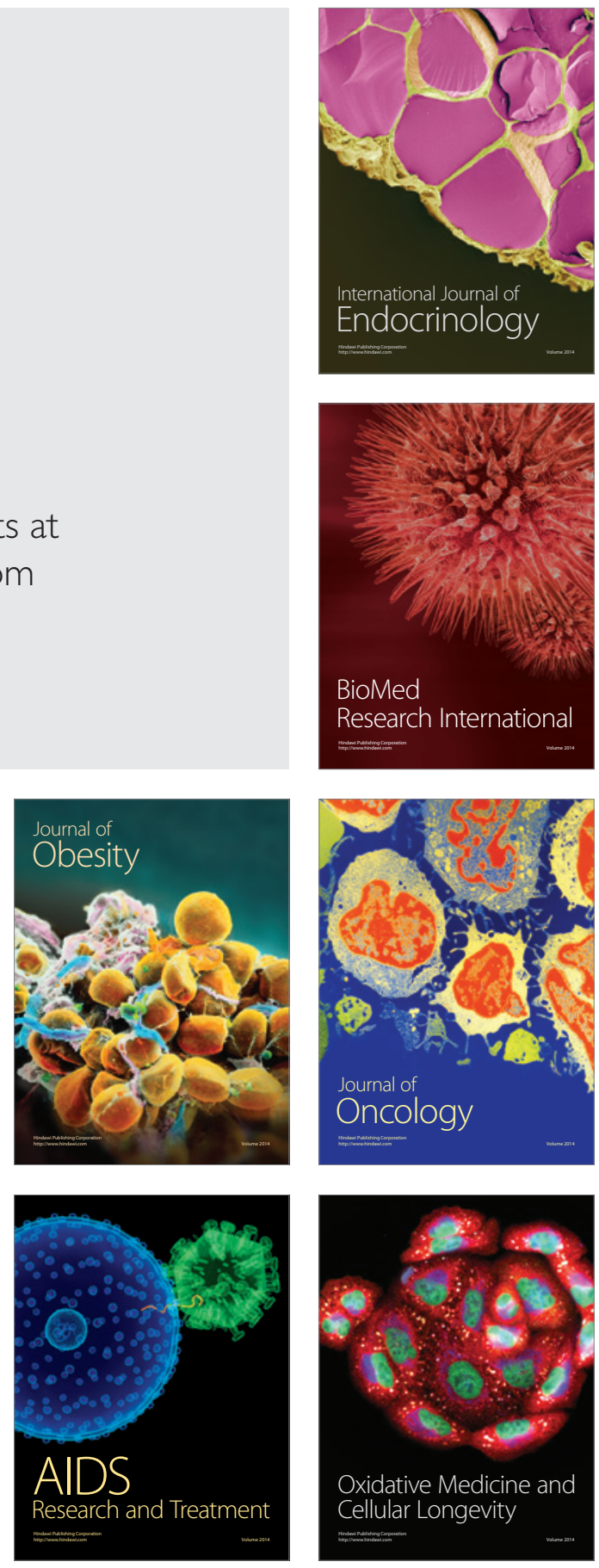